\title{
A comparative study on the morphology, radiography and computed tomography of the skull bones of barking deer (Muntiacus muntjak) and sambar deer (Rusa unicolor)
}

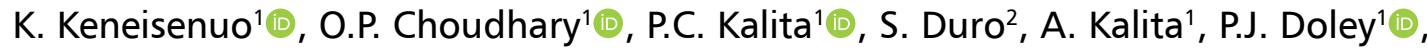 \\ R.S. Arya ${ }^{3}$, S. Debroy ${ }^{1}$, P. Priyanka ${ }^{4} \odot$ \\ ${ }^{1}$ Department of Veterinary Anatomy and Histology, College of Veterinary Sciences and Animal Husbandry, \\ Central Agricultural University (I), Selesih, Aizawl, Mizoram, India \\ ${ }^{2}$ Faculty of Veterinary Medicine, Agricultural University of Tirana, Tirana, Albania \\ ${ }^{3}$ Department of Veterinary Pathology, College of Veterinary Sciences and Animal Husbandry, \\ Central Agricultural University (I), Selesih, Aizawl, Mizoram, India \\ ${ }^{4}$ Department of Veterinary Microbiology, College of Veterinary Sciences and Animal Husbandry, \\ Central Agricultural University (I), Jalukie, Peren, Nagaland, India
}

[Received: 15 December 2020; Accepted: 19 January 2021; Early publication date: 9 February 2021]

Background: There is scanty information on the skull morphology of barking and sambar deer; thus the present study was designed to provide information on morphology, radiography and computed tomography (CT) of the skull bones of both deer species. Materials and methods: The study was conducted on 12 skulls of adult barking deer $(n=6)$ and sambar deer $(n=6)$ of either sex $(n=3$ males and $n=3 \mathrm{fe}$ males) collected from Aizawl Zoological Park, Aizawl, Mizoram. The skulls of both species were macerated as per the standard maceration techniques.

Results: The skull bones of both deer species were divided into a neurocranium and a viscerocranium. The neurocranium was comprised of occipital, sphenoid, temporal, frontal, parietal, interparietal and ethmoid bones. The viscerocranium consisted of nasal, lacrimal, zygomatic, maxilla, incisive, palatine, pterygoid, vomer, mandible, turbinates and hyoid bones. The cranial cavity was oval and elongated caudally. The orbit was round, complete in barking deer; however, it was oval, complete in sambar deer. The facial tuberosity was present caudal to infraorbital foramen and dorsally at superior third premolar tooth in barking deer whereas dorsally at the superior first molar tooth in sambar deer. The infraorbital foramina were small, elliptical and placed at the level of the superior first premolar tooth. The alveolus for a canine tooth was present rostrally in the maxilla of both species. Turbinates bones were visible and mandibular symphysis remained unossified on radiographs and CT in both species. The radiographs of both species showed that the nasal canal was divided by the nasal septum. The CT scan demonstrated the paranasal, frontal and maxillary sinuses. Conclusions: The present study is important in the comparative anatomy of ruminant species and may help the wildlife forensic officials to identify and differentiate the bones of these two species from those of other domestic and wild small ruminants. (Folia Morphol 2022; 81, 1: 164-174)

Key words: barking deer, sambar deer, skulls, morphology, radiography, computed tomography 


\section{INTRODUCTION}

The barking deer, also recognised as Indian Muntjac (Muntiacus muntjak) is a cervid species indigenous to South and South-east Asia and has been classified on the International Union for Conservation of Nature (IUCN) red-list as 'Least concern' [22]; and is protected under Schedule III of Indian Wildlife Protection Act, 1972. This species is named barking deer due to its bark-like sound [1, 13]. Among all the signals used in intra-specific and inter-specific communication, vocalisation has been the primary means of communication for the species living in dense forest habitats [17]. They are moderately small tropical deer with a solitary lifestyle $[1,11,14]$ and extensive natural distribution, ranging throughout a large part of Southeast Asia [11, 16]. The body length of muntjacs varies from 89 to $135 \mathrm{~cm}$ long and their height ranges from 38 to $66 \mathrm{~cm}$ tall with an average weight of 22 to $16 \mathrm{~kg}$ [7]. They are regarded as the most primitive of all living cervidae [15]. The male of barking deer has small antlers projected from the long body hair enveloped pedicles above their eyes, whereas the female has tiny bony knobs. The barking deer may kill the prey by hitting with forelegs and biting using their canines [9].

The sambar deer, also known as sunda sambar (Rusa unicolor) is a large cervid species that is indigenous to the Indian subcontinent, Southern China, and Southeast Asia [12]. This deer species has been categorised by the IUCN red-list as 'vulnerable' due to a decrease in their population year by year [10, 23] and is protected under Schedule-III of the Indian Wildlife Protection Act, 1972. In general, they attain a height of $100-160 \mathrm{~cm}$ at the shoulder and may weigh 100 to $550 \mathrm{~kg}$. The head and body length of sambar deer varies from $162-270 \mathrm{~cm}$, with a $22-35 \mathrm{~cm}$ tail [2]. They inhabit both gentle sloping and steep forested hillsides. They preferably reside near cultivated areas such as gardens and plantations, thick forests, swamp forests and move between higher altitudes during summer and lower, more sheltered areas in winter $[15,18]$.

Several studies on gross morphology, radiography and computed tomography (CT) of skull bones have been undertaken in many domestic and wild small ruminant species such as goats $[3,20]$, sheep $[6,8,21]$ and deer species, i.e. chital, blackbuck and chinkara $[4,5,19]$ in an attempt to give baseline anatomical information.
There is scanty information on the morphology, radiography and CT of skull bones of barking deer and sambar deer. Therefore, the present study has been delineated for determining the morphology, radiography and CT of skull bones of barking and sambar deer.

\section{MATERIALS AND METHODS}

The present study was conducted at Aizawl from March 2019 to June 2020. Twelve skulls of adult barking deer $(n=6)$ and sambar deer $(n=6)$ of either sex ( $n=3$ males and $n=3$ females) were collected. The age of barking deer and sambar deer used for skull collection were in the range of 3 to 4 years and 6 to 8 years, respectively. All procedures involving sample collection were conducted as per the Institutional Animal Ethics Committee (IAEC), College of Veterinary Sciences and Animal Husbandry, Aizawl, Mizoram.

Skulls were collected from the Aizawl Zoological Park, Aizawl, Mizoram, after obtaining official permission from the Principal Chief Conservator of Forest (PCCF) and Chief Wildlife Warden, Government of Mizoram vide letter no. A.22011/5/2017-CWLW/91, dated 15.03.2019. The collected skulls were macerated as per the standard hot water maceration technique [9] after excavating out from the graveyard located at the site of the Aizawl Zoological Park, Aizawl, Mizoram. After maceration, skulls were soaked in $4 \%$ hydrogen peroxide for three days in a sealed container until bones appeared clean and whitish [9]. Finally, processed skulls were sun-dried for three days and utilised for the studies.

The gross morphological studies on skull bones were carried out at the Department of Veterinary Anatomy and Histology, College of Veterinary Sciences and Animal Husbandry, Selesih, Aizawl and Aizawl Zoological Park under the supervision of the zoo officials. The skull bones radiography was carried out by the Allengers-325 X-ray machine (200 mA) and CT scan by Siemens Somatom Emotion instrument (270 mA) at Diagnostic Division Radiology and Imaging, Mizoram Health Care, Aizawl, Mizoram.

The present study was designed to provide information on the morphological, radiographic and CT of the barking and sambar deer skull and provide baseline morphological information. The obtained morphological results of both species were compared with the other domestic and wild small ruminants as per the available literature. 


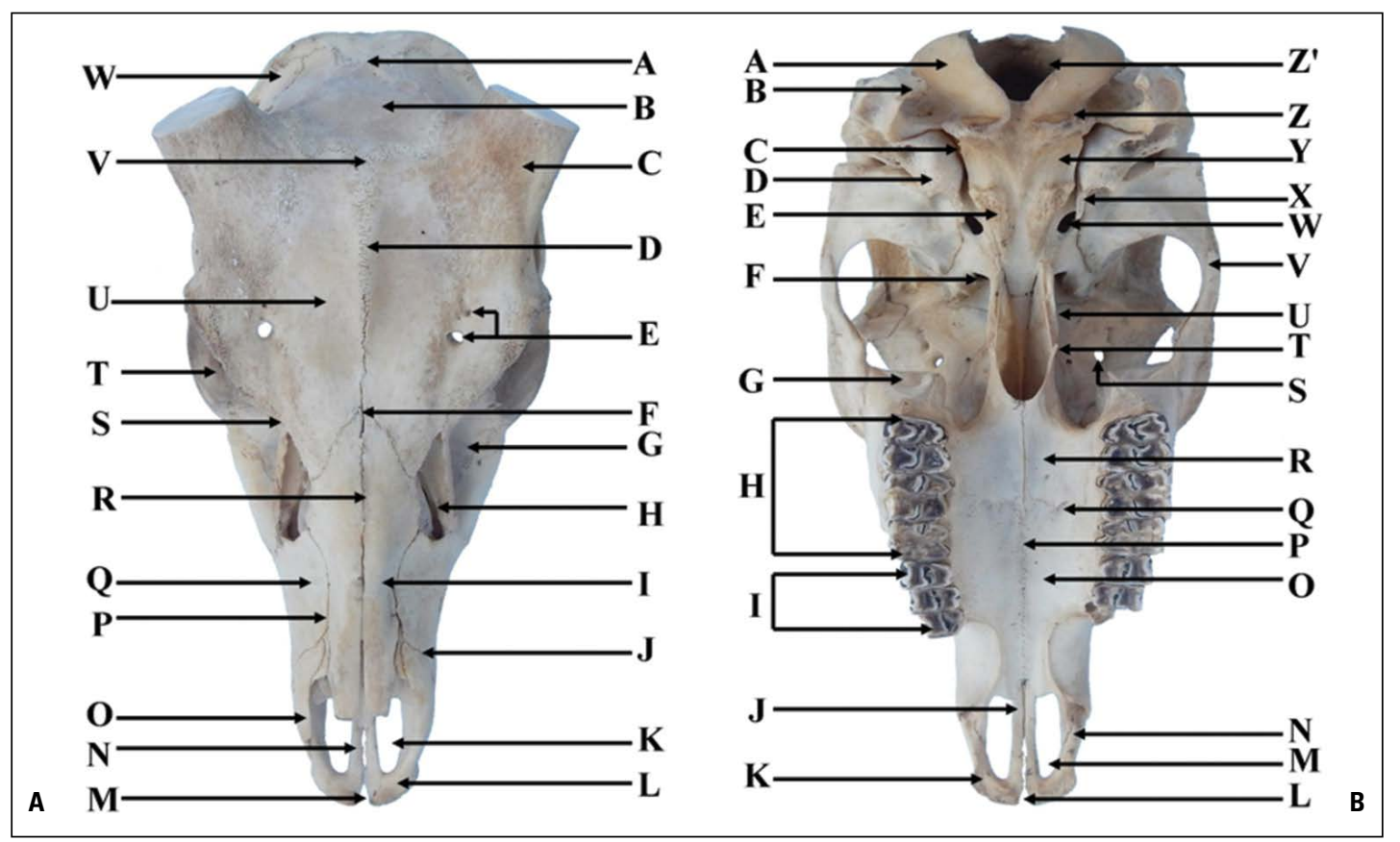

Figure 1. A. Dorsal view of the skull of male sambar deer showing lambdoid suture (A); interparietal bone (B); cornual process (C); interfrontal suture $(D)$; supraorbital foramen $(E)$; nasofrontal suture $(F)$; preorbital fossa $(G)$; nasolacrimal fissure $(H)$; nasal bone $(\mathrm{I})$; maxilloincisive suture $(\mathrm{J})$; palatine fissure $(\mathrm{K})$; body of incisive bone $(\mathrm{L})$; interincisive fissure $(\mathrm{M})$; palatine process of incisive bone (N); nasal process of incisive bone $(0)$; nasomaxillary suture $(P)$; maxilla bone $(0)$; internasal suture $(R)$; frontolacrimal suture $(S)$; orbit $(T)$; frontal bone $(U)$; frontoparietal suture (V); parietal (W); B. Ventral view of the skull of sambar deer showing occipital condyle (A); paracondylar or paramastoid process (B); jugular foramen (C); tympanic bulla (D); muscular tubercle $(E)$; foramen orbitorotundum $(F)$; lacrimal bulla $(G)$; superior first, superior second and superior third molar tooth $(\mathrm{H})$; superior first, superior second and superior third premolar tooth (I); palatine process of incisive bone $(\mathrm{J})$; body of the incisive bone (K); interincisive fissure (L); palatine fissure (M); nasal process of incisive bone (N); maxillary process of palatine bone $(0)$; median palatine suture $(P)$; major palatine foramen $(0)$; horizontal part of palatine bone $(R)$, supraorbital foramen $(S)$; pterygoid hamulus (T); pterygoid bone $(\mathrm{U})$; zygomatic arch $(\mathrm{V})$; foramen ovale $(\mathrm{W})$; muscular process $(\mathrm{X})$; basilar part of occipital bone $(\mathrm{Y})$; ventral condyloid fossa (Z); foramen magnum (Z').

\section{RESULTS}

The skulls of barking and sambar deer (Fig. 1A, B) were elongated and dolichocephalic. The skull bones of both deer species were divided into a neurocranium and a viscerocranium. The neurocranium was comprised of occipital, sphenoid, temporal, frontal, parietal, interparietal and ethmoid bones. The viscerocranium consisted of nasal, lacrimal, zygomatic, maxilla, incisive, palatine, pterygoid, vomer, mandible, turbinates and hyoid bones.

The morphology of individual skull bones (Fig. 1-7A, B) of both species has been compared as per the literature available for other domestic and wild small ruminants. The interspecies comparison has elaborated in the manuscript's discussion section.

\section{DISCUSSION}

\section{Skull as a whole}

The dorsal surface of the skull in both species was formed by parietal, interparietal, frontal, nasal and incisive bones as reported earlier in kagani goat [20], chital [19] and blackbuck [4].

While viewing from the lateral surface, the zygomatic process of temporal bone did not join with the zygomatic process of the frontal bone in both species as mentioned in chital [19], blackbuck [4] and Bardhoka sheep [6].

The orbit was located rostrolaterally and composed of frontal bone dorso-caudally with zygomatic bone ventro-caudally and lacrimal bone rostrally. It was round and complete in barking deer as mentioned earlier in goat of Mizoram [3]. The orbit was complete and oval in sambar deer as reported in chinkara [20]. The greatest contribution in the formation of bony orbit was by frontal bones as compared to zygomatic and lacrimal bones as mentioned in kagani goat [20], chital [19], blackbuck [4], chinkara [20] and Bardhoka sheep [6]. The orbital rim was circular as reported earlier in blackbuck [4]. The orbit joined with the cranial cavity via ethmoidal foramen, optic 

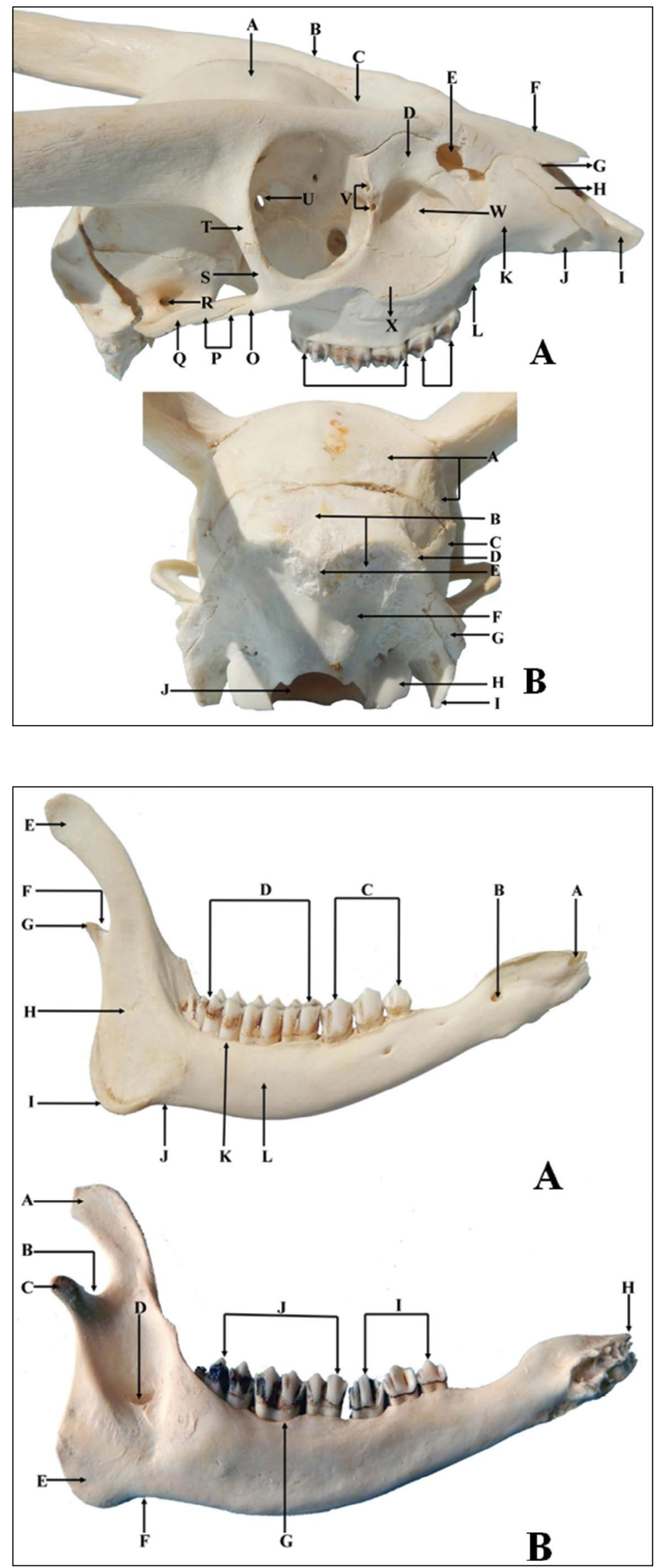

Figure 2. A. Dorsolateral view of the skull of male barking deer showing frontal bone $(A)$; frontal crest (B); shallow concavity on rostral part of frontal bone (C); lacrimal bone (D); nasolacrimal fissure $(E)$; nasal bone $(F)$; nasoincisive fissure $(G)$; incisive bone $(\mathrm{H})$; nasal process of incisive bone $(\mathrm{I})$; alveolus for upper canine tooth $(\mathrm{J})$; maxilla bone $(\mathrm{K})$; alveolus for superior first premolar tooth (L); second superior and third superior premolar tooth (M); first superior, second superior and third superior molar tooth $(\mathrm{N})$; temporal process of zygomatic bone (0); zygomatic arch (P); zygomatic process of temporal bone (0); tempora orifice of temporal meatus (R); frontal process of zygomatic bone $(S)$; zygomatic process of frontal bone $(T)$; optic foramen (U); lacrimal foramen (V); preorbital fossa (W); zygomatic bone (X); B. Nuchal view of the skull of male barking deer showing parietal bone (A); squamous part of occipital bone (B); temporal bone (C); nuchal crest (D); external occipital protuberance $(\mathrm{E})$; lateral part of occipital bone $(\mathrm{F})$; mastoid process of temporal bone $(\mathrm{G})$; occipital condyle (H); paracondylar/paramastoid process (I); foramen magnum (J).

Figure 3. A. Lateral view of mandible of barking deer showing alveolar socket for incisors tooth $(A)$; mental foramen $(B)$; inferior first, inferior second and inferior third premolar tooth (C); inferior first, inferior second and inferior third molar tooth (D); coronoid process/head/height of mandible $(\mathrm{E})$; incisures of mandible $(\mathrm{F})$; condyloid process/condylar process $(\mathrm{G})$; ramus of the mandible $(\mathrm{H})$; mandibular angle (I); notch for facial artery and vein $(\mathrm{J})$; alveolar border $(\mathrm{K})$; body of mandible (L); B. Medial view of mandible of barking deer showing coronoid process/head/ /height of mandible (A); incisure of mandible (B); condyloid process/condylar process (C); mandibular foramen (D); mandibular angle (E); notch for facial artery and nerve (F); alveolar border (G); alveolar socket for incisors tooth $(\mathrm{H})$; inferior first, inferior second and inferior third premolar tooth (I); inferior first, inferior second and inferior third molar tooth $(\mathrm{J})$. 

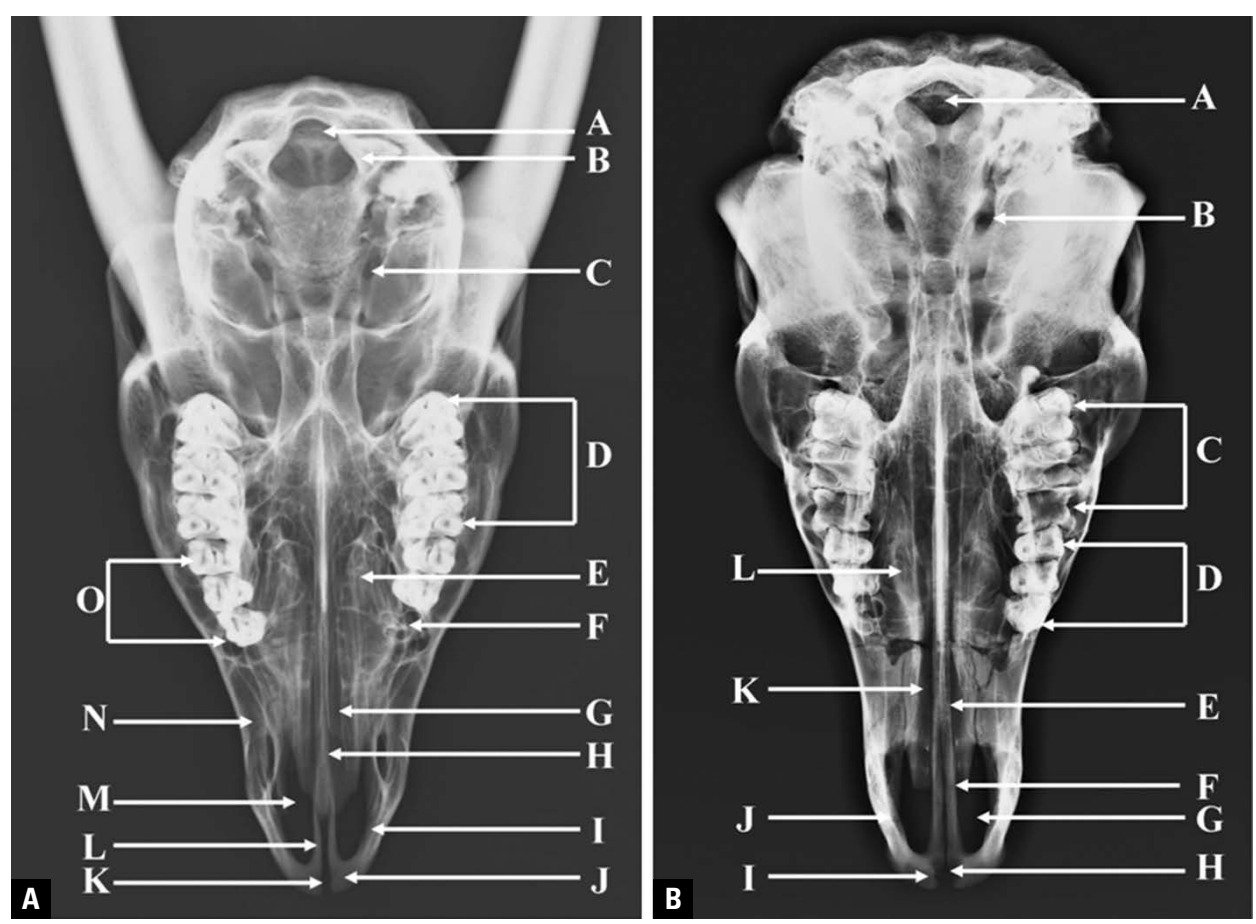

Figure 4. A. Radiograph of dorsal view of the skull of male barking deer showing foramen magnum (A); occipital condyle (B); foramen ovale (C); superior first, superior second and superior third molar tooth (D); turbinate bone (E); alveolar socket of superior first premolar tooth (F); nasal canal $(\mathrm{G})$; vomer bone $(\mathrm{H})$; nasal process of incisive bone $(\mathrm{I})$; body of incisive bone $(\mathrm{J})$; interincisive fissure $(\mathrm{K})$; palatine process of incisive bone (L); palatine fissure (M); pre-orbital fossa (N); superior first, superior second and superior third premolar tooth (0); B. Radiograph of dorsal view of the skull of male sambar deer showing foramen magnum (A); foramen ovale (B); superior first, superior second and superior third molar tooth (C); superior first, superior second and superior third premolar tooth (D); vomer bone (E); palatine process of incisive bone $(\mathrm{F})$; palatine fissure $(\mathrm{G})$; interincisive fissure $(\mathrm{H})$; body of incisive bone $(\mathrm{I})$; nasal process of incisive bone $(\mathrm{J})$; nasal canal $(\mathrm{K})$; turbinate bone (L).

foramen and foramen orbitorotundum as in chital [19], blackbuck [4] and chinkara [20].

In both species, the cranial cavity was ovoid and elongated caudally as mentioned in blackbuck [4] and goat [3]. The cranial cavity roof was formed by the frontal, occipital, interparietal and parietal bones. The floor was made up of the basioccipital and sphenoid bones. The cranial cavity's lateral wall was constituted by occipital, parietal, temporal and frontal bones in the barking and sambar deer as reported earlier in chital [19] and blackbuck [4]. The nasal cavity wall was made up of a cribriform plate of the ethmoid that separated the nasal cavity from the cranium, which was even noticed in chital [19] and blackbuck [4]. A cerebral fossa was noticed dorso-lateral to internal acoustic meatus as reported in chital [19] and blackbuck [4].

In barking and sambar deer, the nasal cavity was a longitudinal passage that expanded via the upper part of facial bone. This cavity was separated into two halves: right and left by median septum nasi as recorded earlier in chital [19] and blackbuck [4]. The lateral wall was formed by the maxilla, incisive and perpendicular part of palatine bones as reported earlier in chital [19] and blackbuck [4]. The nasal cavity floor was lengthy compared to its roof as mentioned earlier in blackbuck [4].

The total numbers of teeth in barking and sambar deer were 34 with an upper canine tooth in maxilla bone. The upper jaw carried the superior 3 premolars and molars and the lower jaw presented 3 incisors, 1 canine, 3 inferior premolars and 3 molars, respectively. However, the total numbers of teeth were 32 due to the absence of an upper canine tooth in chital [19] and blackbuck [4].

\section{Neurocranium}

\section{Os occipitale}

The occipital bone was observed in the caudal surface of the skull in both species and consisted of basilar part, squamous part and lateral parts as reported earlier in chital [19], blackbuck [4] and chinkara [20]. The basilar part was short and wide in barking and sambar deer as described in blackbuck [4]. The 

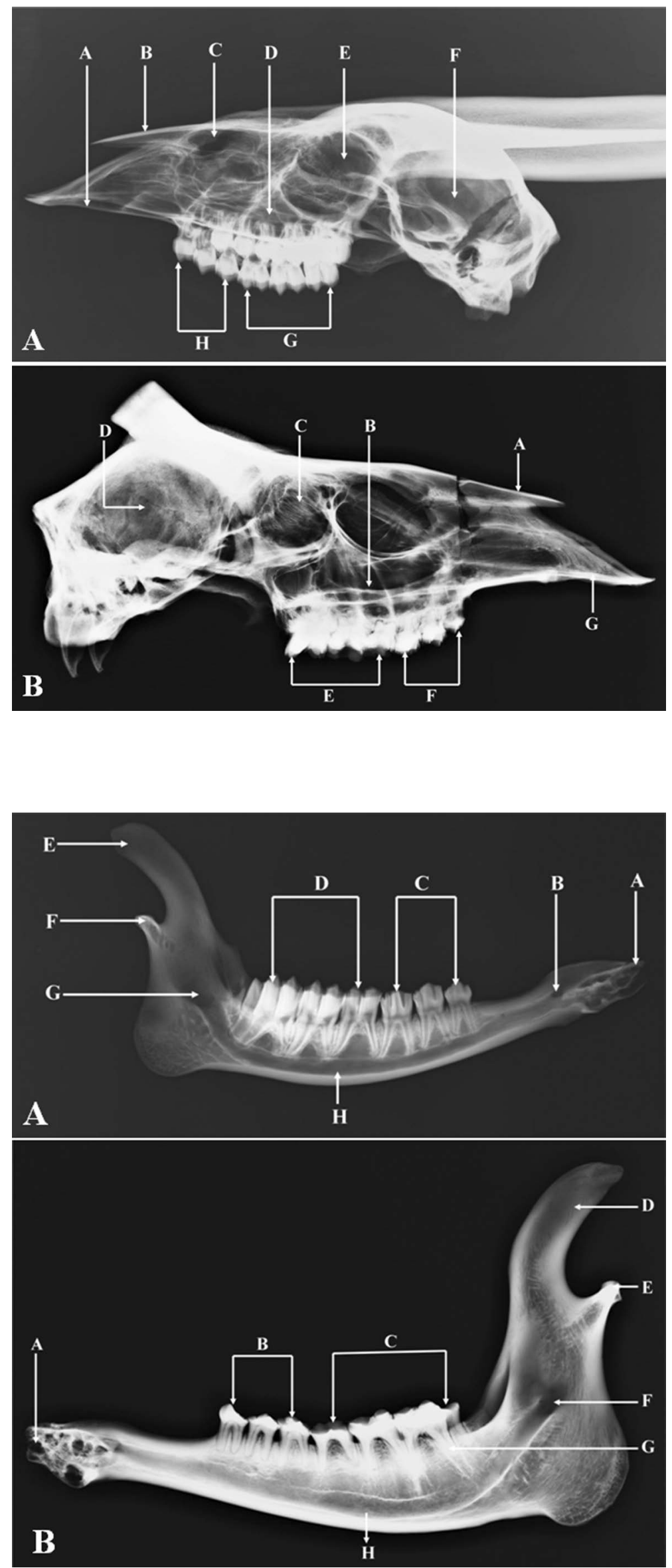

Figure 5. A. Radiograph of lateral view of the skull of male barking deer showing incisive bone $(\mathrm{A})$; nasal bone $(\mathrm{B})$; turbinate bone $(\mathrm{C})$; maxillary sinus (D); orbit (E); cranial cavity (F); superior first, superior second and superior third molar tooth (G); superior first, superior second and superior third premolar tooth $(\mathrm{H})$; B. Radiograph of lateral view of the skull of male sambar deer showing nasal bone $(A)$; maxillary sinus $(B)$; orbit (C); cranial cavity (D); superior first, superior second and superior third molar tooth $(\mathrm{E})$; superior first, superior second and superior third premolar tooth $(F)$; incisive bone $(G)$.
Figure 6. A. Radiograph of lateral view of the mandible of barking deer showing root of the lateral alveolar tooth $(A)$; mental foramen $(B)$; inferior first, inferior second and inferior third premolar tooth (C); inferior first, inferior second and inferior third molar tooth (D); height/head/coronoid process of mandible (E); condylar process $(\mathrm{F})$; mandibular foramen $(\mathrm{G})$; mandibular canal $(\mathrm{H})$; B. Radiograph of medial view of the mandible of sambar deer showing root of the lateral alveolar tooth (A); inferior first, inferior second and inferior third premolar tooth $(\mathrm{B})$; inferior first, inferior second and inferior third molar tooth (C);

height/head/coronoid process of mandible (D); condylar process $(\mathrm{E})$; mandibular foramen $(\mathrm{F})$; root of teeth (G); mandibular canal $(\mathrm{H})$. 


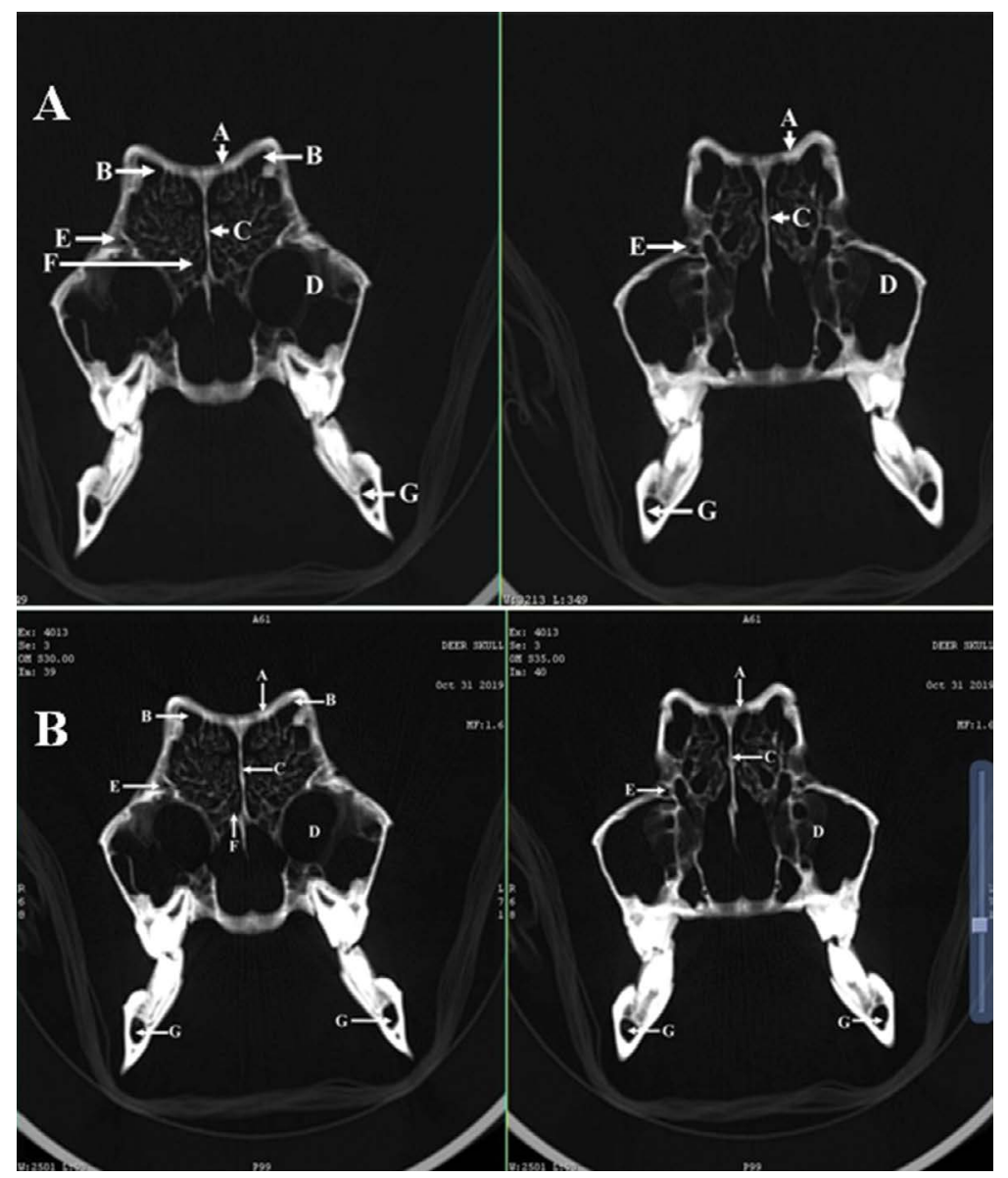

Figure 7. A. Computed tomography scan of the head region of the barking deer showing frontal bone $(A)$, frontal sinus $(B)$, nasal septum (C), maxillary sinus (D), nasolacrimal duct (E), ethmoturbinates with ethmoidal conchae $(F)$ and mandibular canal (G); B. Computed tomography scan of the head region of the sambar deer showing frontal bone $(A)$, frontal sinus $(B)$, nasal septum (C), maxillary sinus (D), nasolacrimal $\operatorname{duct}(E)$, ethmoturbinates with ethmoidal conchae (F) and mandibular canal (G). jugular foramen (Fig. 1B) was located on either side of the basilar part, adjacent to the tympanic bullae. The muscular tubercle was found in the basilar portion of the occipital bone in both species. The squamous part was quadrilateral in both species as reported in blackbuck [4], whereas it was pentagonal in kagani goat [20]. The lateral parts of the occipital bones formed the lateral borders of the foramen magnum in both species. The nuchal crest was less prominent in barking and sambar deer as reported in blackbuck [4]. In both species, the external occipital protuberance was a median triangular projection for attachment of nuchal ligament as noted earlier in chital [19] and blackbuck [4]. The paramastoid processes were thin, prismatic and projected ventrally in barking and sambar deer as noticed earlier in chital [19], blackbuck [4] and chinkara [20]. The lateral parts of the occipital bone formed the lateral borders of the foramen magnum in both species. The lateral parts consisted of occipital condyles with the condylar process. Lateral to the condylar processes, paracondylar processes were present in both species as reported in chital [19] and blackbuck [4]. The hypoglossal canal (canalis nervi hypoglossi) was located between the paracondylar and condylar processes in both species. The foramen magnum was huge and almost oval as mentioned in chital [19], goat of Mizoram [3], chinkara [20] and Bardhoka sheep [6].

\section{Os sphenoidale}

In barking and sambar deer, the sphenoid bone was single. It was located between the basilar portion of the occipital bone and ethmoid bone rostrally as reported in chital [19] and blackbuck [4]. It formed the cranial cavity floor and was composed of two parts: presphenoid rostrally and postsphenoid caudally. The orbital fissure and round foramen (foramen rotundum) unite to form foramen orbitorotundum as mentioned in chital [19] and blackbuck [4]. The oval foramen was formed completely by the sphenoid bone as described earlier in blackbuck [4].

\section{Os temporale}

The temporal bone was paired, irregular in shape in both species. The temporal bone formed the lateral wall of the cranium. This bone was situated between 
parietal bone dorsally, frontal bone cranially, sphenoid bone ventrally and occipital bone caudally as reported in chital [19] and blackbuck [4]. These bones consisted of three parts, namely: the squamous (pars squamosa), petrous parts (pars petrosa) and tympanic part (pars typmpanica). The squamous portion of the temporal bone was shell-like and the petrous portion was placed in between the occipital caudally and parietal cranially and was overlapped exteriorly by squamous temporal bone as mentioned in chital [19] and blackbuck [4]. The zygomatic process of temporal bone did not articulate with the zygomatic process of frontal bone as mentioned in goat of Mizoram [3]. The articular tubercle was present; however, it was absent in chital [19]. The hyoid process was short rod-shaped in both species, projected downwards and onwards beneath the external auditory processes. The mastoid process was present in both species but was absent in chital [19] and blackbuck [4]. The tympanic bulla was huge in both species as mentioned in chital [19] and blackbuck [4]. The temporal fossa was deep and well developed in both species as reported earlier in kagani goat [20], blackbuck [4] and Bardhoka sheep [6].

\section{Os frontale}

The frontal bone was paired and irregular and slightly rectangular in both deer species. The caudal portion of the external surface of these bones was convex with the suppressed cranial part, which was also mentioned in chital [19] and blackbuck [4]. In both species, these bones joined with parietal bone at the frontoparietal suture, caudally and rostrally to nasal bones at the nasofrontal suture. The supraorbital foramen was placed in a supraorbital groove near the orbit's medial margin, similar to the findings in chital [19] and blackbuck [4]. However, it was in the supraorbital groove of the medial brim of the orbit in chinkara [20]. The frontal sinus was merged to the frontal bone as noted earlier in chital [19] and blackbuck [4].

\section{Os parietale}

The parietal bone in both species was paired and composed the dorsolateral wall of the cranial cavity. It was bordered by the occipital bone caudally and frontal bone rostrally in both species as reported in chital [19] and blackbuck [4].

\section{Os interparietale}

In barking and sambar deer, the interparietal bone was unpaired, wide, quadrilateral and situated between occipital and parietal bone as reported in chital [19] and blackbuck [4]. It contributed to the formation of the cranial cavity roof as also reported earlier in chinkara [20].

\section{Os ethmoidale}

In both species, the ethmoid bone was single and placed in between the cranial and nasal cavity as noticed in chital [19], blackbuck [4] and chinkara [20]. Ethmoid bones merged with presphenoid caudally, vomer and palatine bones rostro-ventrally and with frontal bones dorso-rostrally, which was parallel to the findings in chital [19], blackbuck [4] and chinkara [20]. It was composed of the cribriform plate, labyrinth and the perpendicular plate as discussed earlier in chital [19] and blackbuck [4]. In both deer species, it carried an ethmoidal foramen placed in an orbital plate of frontal bone as reported earlier in chital [19] and blackbuck [4]. In contrast, the same foramen was located entirely in the frontal bone in chinkara [20].

\section{Viscerocranium \\ Os nasale}

In barking and sambar deer, the nasal bone (Fig. 1A) was pair of bone articulated rostrally with incisive bone and caudally with the frontal bone as reported earlier in chital [19] and blackbuck [4]. In both species, the nasal bones constituted the larger part of the roof of the nasal cavity. The rostral extremity of these bones was separated into two by a notch. The posterior extremity of the paired nasal bone presented with a notch composed of the rostral part of frontal bones resembled the observations in chital [19] and blackbuck [4].

\section{Os lacrimale}

In both species, the lacrimal bone (Fig. 2A) was paired. These bones superiorly articulated with frontal and nasal bones, ventrally with maxilla bone and caudally with the malar bone as described in chital [19]; however, the lacrimal bone connected with frontal bones superiorly, maxilla bones ventrally, malar bones caudally and palatine bone beneath and beyond in blackbuck [4]. The larger and smaller parts of lacrimal bones were combined together along the orbital rim. A lacrimal bulla was noted in both species as described in chital [19] and blackbuck [4]. These bones also presented a deep nasolacrimal fissure in both species as reported in Bardhoka sheep [6], but it was much deeper in sambar deer. In both species, 
very deep and prominent depression or fossa called lacrimal fossa for lodgement of the preorbital gland as described in Bardhoka sheep [6] and these fossae were deeper in sambar deer.

\section{Os zygomaticum}

In both species, the zygomatic bone was paired and situated between lacrimal above and maxilla beneath and in front as reported earlier in blackbuck [4]. These bones were curved crest-like, around the infraorbital margin continuing to the maxilla bone as also reported in chital [19] and blackbuck [4]. The orbital surface of the zygomatic bone was smaller than the lateral surface in both species similar to findings in chital [19] and blackbuck [4]. The frontal process of the zygomatic bones articulated with the zygomatic process of the frontal bones, as mentioned in chital [19] and blackbuck [4].

\section{Os maxillare}

In barking and sambar deer, the maxilla (Fig. 2A, B) was irregular and placed between the nasal and incisive bones. The facial tuberosity was present caudal to the infraorbital foramen as elucidated in blackbuck [4] and Bardhoka sheep breed [6]. This tuberosity was present dorsally at the superior third premolar tooth in barking deer as also discussed in chital [19], goat [3]; and was dorsally at the superior first molar tooth in sambar deer, which was also noted in Bardhoka sheep [6]. However, the same was placed at the junction of fourth and fifth cheek teeth in kagani goat [20] and was at the level of fifth cheek tooth in Mehraban sheep [8]. A small, elliptical infraorbital foramen was placed at the level of the superior first premolar tooth as mentioned in chital [19], while the infraorbital foramen was oval-shaped and placed in maxilla bone dorsally and oriented rostrally at the level of second cheek tooth in chinkara [20] and was located at the level of the first molar in Bardhoka sheep [6]. The facial crest was found in both the deer species; however, it was very prominent in Mehraban sheep [8]. The facial tuberosity was observed dorsally at the superior third premolar in barking deer as reported earlier in chital [19] and goat [3]. In contrast, it was placed dorsally at the superior first molar tooth in the sambar deer, which was just caudal to infraorbital foramen in chital [19], blackbuck [4] and Bardhoka sheep [6]. In other studies, it was found to be at the intersection of fourth and fifth cheek teeth in kagani goat [20], at the level of fifth cheek tooth in sheep [8].
The facial crest caudally extended from facial tuberosity to the facial surface of the zygomatic bone. In both species, the alveolar border presented 6 alveoli for the superior 3 premolar and superior 3 molar as also noted in chital [19] and blackbuck [4]. The palatine process (Processus palatinus) was a thin plate that forms the rostral part of the basis of hard palate as reported in blackbuck [4].

\section{Os incisivum}

In both species, the incisive bone (Fig. 2A) was a paired bone, thin and wide plate as also reported in chital [19]. It did not carry any alveolus for upper teeth and presented a dental pad as in chital [19] and blackbuck [4]. The nasal process of incisive bone was joined to the nasal bone. The palatine process was a thin plate that composed the rostral part of the basis of the hard palate in barking and sambar deer, which was also recorded in chital [19] and blackbuck [4]. The palatine fissure was wide and elongated. Both species also presented nasoincisive incisure as also noticed in Bardhoka sheep [6].

\section{Os palatinum}

In the barking and sambar deer, the palatine bone (Fig. 1B) was a paired bone and visualised in the ventral surface of the skull between the maxilla, sphenoid and pterygoid bones. The horizontal plate of palatine bones was extensive, whereas the same was narrow in chital [19] and blackbuck [4]. The two major palatine foramina were perforated on the palatine bone as reported earlier in chital [19] and blackbuck [4]. In contrast, it was placed on the intersection of the horizontal plate of palatine bone and the palatine process of the maxilla in chinkara [20]. In both deer species, the transverse palatine suture was " $\mathrm{V}$ " shaped and serrated, lying over the greater palatine foramina as reported in kagani goat [20].

\section{Vomer}

In barking and sambar deer, the vomer was an unpaired bone. It remained fused with the incisive, maxilla and sphenoid bone and did not articulate with the palatine bone as mentioned in chital [19] and blackbuck [4]. The cranial third of these bones fitted into the nasal crest of the maxilla in both species that was also recorded in chital [19] and blackbuck [4]. However, the laminae of the vomer bone terminated at the junction of palatine processes of maxilla and palatine processes of incisive bones in Bardhoka sheep [6]. 


\section{Os pterygoideum}

In both species, the pterygoid was a paired bone. These bones (Fig. 1B) were thin bony plates and placed between the sphenoid and horizontal plate of the palatine bone. The pterygoid hamulus in both species was a hook-shaped process as reported in chital [19] and blackbuck [4].

\section{Mandibula}

In both species, the mandible was a paired bone consisted of a body and a ramus. The mandibular symphysis (Fig. 3; 6A, B) remained unossified as mentioned in chital [19] and blackbuck [4]. The medial surface of the mandibular symphysis displayed large interdigitating septa in both species that entered into large mediolateral depressions of the opposing mandible. The mandibular corpus was moderately curved when placed on a flat surface and did not join the surface at each extremity. In barking and sambar deer, an incisive part of the body remained elevated from the ground, resembling to chital [19] and blackbuck [4]. The mandible also presented 6 alveoli for inferior 3 premolar teeth and inferior 3 molar teeth in both species as reported in chital [19] and blackbuck [4]. The mandible also presented 8 alveoli for canine and incisors as mentioned earlier in chital [19]. In both deer species, the mental foramen, an external opening of the mandibular canal was located in the fossa placed at junction with the body of the mandible as stated in chital and blackbuck [4, 19]. The mental foramina in sambar deer were exceptionally large and in most cases elongated but were complete spheres in the barking deer. The mandibular foramina were oval or elongated in sambar and barking deer. The mandibular tuberosity was absent, which was also similar to chital [19]. The caudal and perpendicular part of the mandible (ramus) was the non-tooth bearer part as reported in chinkara and [20] blackbuck [4]. In the barking and sambar deer, the articular extremity or caudal extremity contained the condylar process and coronoid process with an intervening notch. The coronoid process was curved caudally and caudodorsally expanded above the condyle and formed the tallest point of the bone that was the same as observed in chital [19] and blackbuck [4]. The head of the condylar process of the mandible was transversely elongated as reported earlier in chital [19] and blackbuck [4].

\section{Ossa turbinata}

In barking and sambar deer, the turbinate bones (Fig. 4A, B) were paired, very fine, scroll-like, complex bony plates located vertically in the nasal cavity attached to the lateral wall of the nasal cavity. These bones arose from dorsal and lateral walls of ethmoid bones in both deer species. Turbinate bones were visible on radiographs and $\mathrm{CT}$ in both species. There were paired dorsal and paired ventral turbinates on each edge of the nasal cavity as recorded in chital [19] and blackbuck [4].

\section{Os hyoideum}

The hyoid bone was an unpaired bone and its morphology was similar to the domestic and wild small ruminant species such as goat of Mizoram, chital and blackbuck $[3,4,19]$.

\section{Radiographic study}

The radiograph of skull bones of barking and sambar deer (Fig. 4-6A, B) showed the dolichocephalic type and elongated as mentioned in blackbuck [4]. The nasal canal was divided by a nasal septum similar to chital [19]. The cranial cavity was visibly recognised with the foramen magnum located along its caudoventrally as reported in chital [19]. The ventrodorsal aspect was more appropriate for the vomer, nasal septum, nasal cavity, premolar and molar teeth. The dorsoventral view showed better visualisation of the vomer, palatine and maxillary sinuses that was also noticed in blackbuck [4]. The occipital condyles and foramen magnum were also clearly recognized in both species as reported in sheep [21] and blackbuck [4].

\section{Computed tomography study}

The CT (Fig. 7A, B) scan of skull bones of both species clearly showed the paranasal, frontal and, maxillary sinuses. A thick bony septum (nasal septum) completely separated the left and right frontal sinus, and a thin incomplete bony septum divided the maxillary sinus into rostral and caudal compartments as was recorded in chital [19].

The frontal sinuses of both species were roughly triangular in outline with its base located medially and the same was also reported in chital [19]. These sinuses were air-filled cavities formed by the evagination of frontal bones into the nasal cavity. The frontal sinus was bounded by frontal bone dorsally, medial wall of the orbit ventrolaterally, cranium caudoventrally 
and turbinates rostroventrally and continued directly to the caudal maxillary side. A thick midline bony septum (nasal septum) completely divided both left and right frontal sinuses as also mentioned in chital [19]. The frontal sinus presents rostral and caudal compartments extended to the cornual processes in barking and sambar deer.

In both species, the maxillary sinuses were an air-filled cavity formed by the evagination of maxillary, lacrimal and zygomatic bones as also reported in chital [19]. These sinuses directly communicated with the nasal cavity. The lateral wall of the maxillary sinus was comprised of the maxilla, lacrimal and zygomatic bones, while the alveolar part of the maxilla formed the floor or ventral wall. The frontal sinus was bounded by the maxilla, ventral turbinate and the lateral mass of ethmoid bone, medially. A thin incomplete bony septum divided the sinuses into two compartments that are cranial and caudal and this septum angled caudally oblique with the varied position in its rostral aspect, generally over the roots of second and third molar teeth and the same was also described in chital [19].

\section{CONCLUSIONS}

The skull morphology derived in the present study can help the wildlife forensic officials, researchers, clinicians and surgeons in giving better knowledge about the different anatomical structures present on the skull bones and its comparative observation between various domestic and wild small ruminants.

\section{Conflict of interest: None declared}

\section{REFERENCES}

1. Adnyane IKM, Zuki ABZ, Noordin MM, et al. Morphological study of the infraorbital gland of the male barking deer, muntiacus muntjak. African J Biotech. 2011; 10(77): 17891-17897, doi: 10.5897/ajb10.2634.

2. Boitani L. Simon \& Schuster's guide to mammals. Simon \& Schuster/Touchstone Books, New York, USA 1984.

3. Choudhary OP, Priyanka P, Kalita PC, et al. A morphometrical study on the skull of goat (capra hircus) in mizoram. Int J Morphol. 2020; 38(5): 1473-1478, doi: 10.4067/ s0717-95022020000501473.

4. Choudhary OP, Singh I. Morphogical and radiographic studies on the skull of Indian blackbuck (Antilope cervicapra). Int J Morphol. 2016; 16(2): 775-783, doi: 10.4067/ S0717-95022020000501473.

5. Din S, Masood S, Zaneb H, et al. Gross and Clinical Anatomy of the Skull of Adult Chinkara (Gazella bennettii). Pak J Zool. 2020; 52(5), doi: 10.17582/journal. pjz/20190207070209.
6. Gündemir O, Duro S, Jashari T, et al. A study on morphology and morphometric parameters on skull of the Bardhoka autochthonous sheep breed in Kosovo. Anat Histol Embryol. 2020; 49(3): 365-371, doi: 10.1111/ahe.12538, indexed in Pubmed: 32022341.

7. Ilyas O, Khan JA. Food habits of barking deer (Muntiacus muntjak) and goral (Naemorhedus goral) in Binsar Wildlife Sanctuary, India. Mammalia. 2003; 67(4), doi: 10.1515/ mamm-2003-0406.

8. Karimi I, Onar V, Pazvant G, et al. The cranial morphometric and morphologic characteristics of Mehraban sheep in western Iran. Global Vet. 2011; 6(2): 111-117.

9. Keneisenuo K, Choudhary OP, Kalita PC, et al. Comparative morphometrical studies on the skull bones of barking deer (Muntiacus muntjak) and sambar deer (Rusa unicolor). Anat Histol Embryol. 2021; 50(3): 500-511, doi: 10.1111/ ahe.12653, indexed in Pubmed: 33423310.

10. Leslie D. Rusa unicolor (Artiodactyla: Cervidae). Mammalian Species. 2011; 43: 1-30, doi: 10.1644/871.1.

11. Long JL. Introduced mammals of the world: their history, distribution and influence. CSIRO Publishing, Collingwood Victoria, Australia 2003.

12. Medway L. The wild mammals of Malaya. Oxford University Press, London, UK 1969.

13. Miazi OF, Miah G, Bilkis T, et al. Phenotypic and reproductive parameters of barking deer under management condition of chittagong zoo. Int J Genet Genomics. 2016; 4(5): 40-44, doi: 10.11648/j.ijgg.20160405.11.

14. Mishra HR. The ecology and behaviour of chital (Axis axis) in the Royal Chitawan National Park, Nepal with comparative studies of hog deer (Axis porcinus), sambar (Cervus unicolor) and barking deer (Muntiacus muntjak). Ph.D. Thesis, Edinburgh, University, Edinburgh, 1982.

15. Nowak RM. Walker's Mammals of the world. 6th edn. The Johns Hopkins University Press, Baltimore, London, UK 1999.

16. Ohtaishi N, Gao Y. A review of the distribution of all species of deer (Tragulidae, Moschidae and Cervidae) in China. Mammal Rev. 1990; 20(2-3): 125-144, doi: 10.1111/j.1365-2907.1990.tb00108.x.

17. Oli MK, Jacobson HA. Vocalizations of barking deer (Muntiacus muntjak) in Nepal. Mammalia. 1995; 59(2), doi: 10.1515/mamm.1995.59.2.179.

18. Payne J, Francis C, Phillips KA. A field guide to the mammals of Borneo. The Sabah Society with WWF Malaysia, Malaysia 1985.

19. Ramswarup. Gross anatomical studies on the bones of the skull in chital (Axis axis). M.V.Sc. Thesis. Rajasthan University of Veterinary and Animal Sciences, Bikaner, Rajasthan, India, 2011.

20. Sarma K. Morphological and Craniometrical Studies on the Skull of Kagani Goat (Capra hircus) of Jammu Region. Int J Morphol. 2006; 24(3), doi: 10.4067/s071795022006000400025.

21. Sarma K. Suri S Kalita A. Gross anatomical studies on the skull of local sheep of Jammu region. Indian J Anim Sci. 2007; 77(10): 999-1001.

22. Timmins RJ, Duckworth JW, Hedges S. Muntiacus muntjak. The IUCN red list of threatened species. International Union for Conservation of Nature and Natural Resources, 2016.

23. Timmins RJ, Kawanishi K, Giman B, et al. Rusa unicolor. The IUCN red list of threatened species. IUCN, 2015. 